ज्ञ FRANÇAISE

$\supset \mathrm{DE}$

될 PÉDAGOGIE

\section{Revue française de pédagogie}

Recherches en éducation

154 | janvier-mars 2006

La construction des politiques d'éducation : de nouveaux rapports entre science et politique

\title{
Représentations du volley-ball scolaire et genre des élèves : pertinence de l'inventaire des rôles de sexe de Bem?
}

School volleyball representations and students' gender: How relevant is Bem sex role inventory?

Vorstellungen des Volleyballs in der Schule: Relevanz des Inventars der Geschlechtsrollen von Bem?

Representaciones del voleibol escolar y género de los alumnos : ¿pertinencia del inventario de los papeles de género de Bem?

Ingrid Verscheure, Chantal Amade-Escot et Catherine-Marie Chiocca

\section{OpenEdition \\ Journals}

Édition électronique

URL : http://journals.openedition.org/rfp/153

DOI : $10.4000 /$ rfp. 153

ISSN : 2105-2913

Éditeur

ENS Éditions

Édition imprimée

Date de publication : 1 mars 2006

Pagination : $125-144$

ISBN : 2-7342-1039

ISSN : 0556-7807

\section{Référence électronique}

Ingrid Verscheure, Chantal Amade-Escot et Catherine-Marie Chiocca, « Représentations du volley-ball scolaire et genre des élèves : pertinence de l'inventaire des rôles de sexe de Bem ? ", Revue française de pédagogie [En ligne], 154 I janvier-mars 2006, mis en ligne le 01 mars 2010, consulté le 01 mai 2019. URL : http://journals.openedition.org/rfp/153; DOI : 10.4000/rfp.153 


\title{
Représentations \\ du volley-b dl scolaire et genre des élèves: pertinence de l'inventaire des rôles de sexe de Bem?
}

\author{
Ingrid Verscheure \\ Chantal A made-EScot \\ C a the rine-M a rie Chiocca
}

L'objet de cet article est de discuter la pertinence de l'"Inventaire des rôles de sexe de Bem"dans sa version courte traduite par Fontayne. Sarazin et Famose en tant qu'outil méthodologique. Dans le cadre d'une enquête en lycées agricoles nous avons étudié les liens entre trois variables : sexe, genre (établi par l'IRSB) et représentations du volley-ball scolaire (à partir d'association de mots et de réponses à un différenciateur sémantique inspiré d'Osgood). Les résultats mettent en évidence que seule la variable sexe contribue significativement aux modalités représentationnelles des élèves. Les modalités de la variable genre (féminin, masculin, androgyne et non différencié, construites par l'IRSB) ne semblent avoir que très peu de liens sauf pour quelques rares modalités de réponse avec les représentations. Ces résultats, au premier abord paradoxaux sont discutés dans une perspective de réflexion théorique et méthodologique autour de la "mesure du genre" dans les recherches en éducation.

Descripteurs (TEE) : Comportement de l'élève, différence de sexe, éducation physique et sportive, rôle de l'enseignant, volley-ball.

C et article examine les relations existant entre le sexe, l'orientation de genre et les représenta tions qu'ont les lycéennes et les lycéens vis-à-vis du volley-ball en tant qu'activité scolaire. L'hypothèse initiale était d'étudier ces trois variables et leurs éventuels liens avec le rapport des élèves à l'activité physique enseignée, ici le volley-ball, et ainsi d'éclairer les processus différentiels d'apprentissage entre filles et garçons. Plusieurs auteurs ont montré l'impact des représentations des sports Collectifs sur les processus didactiques et pointés Certaines disparités selon le sexe (David, 1995 ; Davisse, 1999 ;
Tanguy, 1992). Nous souhaitions approfondir cette question et postulions à la suite de travaux de Fontayne, Sarrazin et Famose (2001 \& 2002) que la perception que les adolescentes et les adolescents accordent aux APS proposées en éducation physique devait être liée, au-delà de la variable sexe, avec le genre en tant qu'intériorisation des rôles sociaux sexuellement typés. Pour valider cette hypothèse une enquête par questionnaire a été sou$m$ ise aux élèves des lycées agricoles de la région Midi-Pyrénées. Dans le cadre de cet article, nous n'aborderons que les résultats relatifs aux relations 
existant entre les variables sexe, genre - Construite à partir de l'Inventaire des rôles de sexe de Bem (IRSB) (1) - et les représentations sociales et fonctionnelles du volley-b a.

Les résultats de l'enquête ont paradoxalement $m$ is en évidence très peu de liens entre les modalités de genre/IRSB (féminin, masculin, androgyne et non différencié) et les représentations du volley-b a des élèves interrogés. Ceci nous a amenées à discuter tout au long de cet article la pertinence de l'outil psychométrique utilisé. Après avoir présenté le Contexte et le cadre théorique de l'étude, précisé les éléments de méthodes et résumé les principaux résultats, nous discutons de l'utilisation de l'IRSB pour les recherches en éducation à la lumière d'une réflexion sur la notion d'orientation de genre dans sa dimension anthropologique.

\section{CONTEX TEE LA RECHERCHE ET CADRE THÉORIQUE}

Dans le cadre d'une recherche didactique portant sur la construction de la différence des sexes en EPS (Verscheure, 2005) nous nous sommes interrogée sur les interactions entre "orientation de genre" et "représentations des APS»dont la littérature a montré le rôle dans les processus d'enseignementapprentissage. Nous pensons - avec d'autres - que "l'orientation de genre" en tant que construction sociale et Culturelle de "modèles intégrés de traits, de comportements, de tâches et d'activités pouvant être endossés ou non par les individus" (Marro, 2003) détermine en partie les représentations qu'ont les élèves des activités scolaires que les institutions (didactiques ou non) leur proposent. S'il peut être décrit, Ce lien serait susceptible d'expliquer Certains phénomènes différentiels à l'œuvre dans les situations d'enseignement-apprentissage. Ce postulat rejoint le point de vue développé par Mosconi (2003) dans le cadre des travaux de son équipe sur le rapport au savoir socio-sexué à l'école. Notre interrogation s'enracine également dans les résultats d'études exploratoires ayant $m$ is en évidence que les "positions de genre" des élèves s'actualisent en classe du fait des effets Conjugués des Contextes d'apprentissages (tâches scolaires proposées aux élèves), des interactions didactiques avec l'enseignant et les autres élèves, elles-mêmes influencées par les attitudes plus ou moins scolaires et les arrière-plans représentationnels (2) (Amade-Escot, 2005 ; Verscheure \& Am ade-Es Cot, 2004 ) sur lesquels s'élabore, se modifie ou se "Colore" l'activité d'apprentissage de l'élève (Sarrazy, 1996). L'étude que nous présentons s'inscrit dans les perspectives ouvertes par deux domaines de recherche encore peu articulés: Celui des inégalités scolaires selon l'orientation de genre, en tant que dimension psychologique et sociale explicative des rapports sexués aux apprentissages scolaires; Celui de la recherche en didactique et du rôle joué par les représentations dans les rapports des élèves aux savoirs enseignés.

\section{Les inégalités de réussite entre filles et garçons en EPS}

Les travaux de sociologie différentielle et de psychologie sociale (pour une revue en langue française : Duru-Bellat, 1994 \& 1995 ; pour une revue en Iangue anglaise : Sadker \& Sadker, 1991), tout Comme les études féministes anglo-saxonnes dans le domaine du sport et de l'éducation physique (pour une revue internationale: Penney, 2002) soulignent l'importance de l'orientation de genre Comme Construction sociale de la différence des sexes et Comme mode d'incorporation des usages, normes, valeurs et représentations sociales attribuées aux filles et aux garçons tout au long de leur scolarité (Durand-Delvigne \& Duru-Bellat, 1998 ; Duveen, 1999). Sans développer ici toutes les discussions et Controverses relatives aux distinctions entre rôle de sexe, identité de genre, stéréotypes sociaux de sexe, etc., nous Considérons que la notion d'orientation de genre a permis de dépasser la perspective naturaliste associée à la différence biologique de sexe (Héritier, 1996) en y pointant la Composante socioculturelle et l'historicité Constitutive de la Construction de la "différence des sexes" (Fraisse, 1996). C'est dans Cette perspective, à la suite des travaux pionniers de Davisse et Louveau (1991), que les recherches portant sur les inégalités sexuées en éducation physique se sont développées dans la période Contemporaine (Cogerino, 2005 ; Davisse, 1999 \& 2000 ; Vigneron, 2004). Ces auteures Considèrent que les inégalités de réussite entre filles et garçons en éducation physique sont minorées en raison d'une part, de la prééminence de la valeur biologique comme explicative des différences de performances entre filles et garçons qui se traduit par des inégalités de notation au baccalauréat (Bergé, 2000 ; Coupey, 1995 ; Vigneron, 2004); et d'autre part, de la non-reconnaissance des formes spécifiques de rapports aux savoirs scolaires selon les identités de genre (Cogerino, 2005 ; Vigneron, 2004). Selon une autre perspective, une étude 
récente met en évidence des engagements différenCiés selon "l'orientation de genre" des filles et des garçons de lycée vis-à-vis de la pratique sportive en pointant le lien entre sexe/filière et pratique volontaire du sport (Vouillot, 2003). Les résultats montrent que les garçons de filières scientifiques sont les plus nombreux à faire du sport (en dehors du temps d'EPS obligatoire) et qu'un certain nombre de filles renoncent simultanément au sport et à l'étude des sciences. Le garçon et la fille sportif/ve/s sont décrits Comme "masculins " et "androgynes " par un grand nombre des sujets interrogés, œ qui amène l'auteure à conclure que "le sport n'est pas associé à la féminité »(Vouillot, 2003).

De l'ensemble de ces travaux il apparaît que I'EPS, en tant que matière d'enseignement, contribue à renforCer et légitimer d'importantes inégalités entre filles et garçons qui se Construisent tout au long du processus interactif $d u$ fait d'une faible prise en Considération des "orientations de genre" (en tant qu'intériorisation des rôles de sexe) et des représentations sexuées des élèves vis-à-vis des activités physiques enseignées.

Parmi les activités physiques enseignées en EPS, les sports Collectifs ont une présence importante dans les programmes scolaires et le volley-ball est très souvent programmé, notamment au lycée. Les recherches ont montré que les sports Collectifs sont assez emblématiques des processus de différentiation sexuée en EPS du fait de leur connotation généralement m as Culine (David, 1995 ; Ennis, 1998 ; Griffin, 1984 ; Vigneron, 2004). Les Conclusions de Ces auteur/e/s rejoignent les réflexions mettant en avant la nécessité de développer des analyses très précises incluant le point de vue didactique en prenant en considération les rapports sexués aux savoirs scolaires Comme variable explicative des différences d'implication et d'acquisition entre filles et garçons (Félix, 1996; Mosconi, 2003). À partir de Ces Considérations il est apparu pertinent de s'intéresser au volley-ball, sport Collectif décrit Comme un sport "approprié aux deux sexes" (Fontayne, Sarrazin \& Famose, 2001,p. 27). Le choix du volleyball permet selon nous d'atténuer les biais liés au marquage sexué des autres sports collectifs Comme activités physiques et sportives (APS) plutôt masculines, notamment du fait d'espaces de jeux séparés par le filet et de l'absence de Contacts entre les opposant/e/s, tout en rendant possible une approche fine des effets de l'orientation de genre sur les perceptions et les représentations des sujets interrogés.

\section{Le statut des représentations en didactique}

Il existe une importante littérature sur la problém atique des relations entre représentation et didactique à partir du postulat selon lequel l'apprentissage est un processus au Cours duquel les représentations se modèlent $p$ ar paliers successifs. La notion, empruntée à la formation d'adultes au début des années 1970 a suscité une vaste littérature en didactique des disciplines dans le but de comprendre le rôle des représentations dans les processus d'apprentissage. Dans les années 1980, en didactique des sciences expérimentales notamment, le terme de Conception s'est progressivement substitué à Celui de représentation en relation avec la question des erreurs et des obstacles. Plus récemment la thématique des représentations a fait l'objet d'une reprise lors d'un dossier thématique s'attachant à dégager la notion de l'arrière-fond mentaliste et intellectualiste qui est souvent le sien, pour insister sur sa dimension processuelle, dynamique et profondément hétérogène et pour pointer la nécessité d'explorer les couplages représentation, activité et situations (Schubauer-Leoni, 2002 ; Sensevy \& Sallaberry, 2002 ).

En didactique de l'éducation physique, la problématique des représentations a fait l'objet d'une importante production de recherches et de controverses actuellement atténuées. Les débats - que nous ne développons pas ici-entre les tenants des approches sociales ou fonCtionnelles des représentations en didactique (Aubert, 1997 ; Bouthier, 1993 ; Bouthier \& David, 1989 ; Bouthier et al., 1990 ; Clément, 1996 ; David, 1995 \& 2001 ) portaient sur la nature des apprentissages visés en éducation physique et l'utilité (ou non) du Concept de représentations pour en rendre Compte. Il apparaît aujourd'hui acquis d'envisager les contributions conjuguées des représentations sociales - souvent sexuellement Connotées (David, 1995) - et des représentations fonctionnelles ou représentations pour l'action, au sens classique de la psychologie du travail (WeillFassina, Rabardel \& Dubois, 1993). Cette position défendue par David (2001) s'appuie sur le fait que les Contenus d'enseignement en éducation physique renvoient à des savoirs et savoir-faire, impliquant des transformations Corporelles, et transposés à $p a r$ tir de pratiques sociales d'activités physiques et sportives ayant diverses connotations sexuées. C'est pourquoi, dans notre étude finalisée par une problématique didactique nous avons exploré les représentations sociales et fonctionnelles des élèves à propos du volley-ball scolaire. 


\section{Questions à l'origine de l'étude}

Faisant l'hypothèse que les représentations des APS jouent un rôle déterminant dans la façon dont les élèves (filles et garçons, en fonction de leur orientation de genre) décodent les signific aions des tâches qui leur sont proposées, notre ambition était d'explorer les liens entre orientation de genre et représentations des élèves, étape préalable à l'étude des dynamiques différentielles de Construction des savoirs en situations d'enseignement-apprentissage du volley-b a (voir Verscheure, 2005).

Nous avons donc onsidéré, à la suite de notre revue de littérature, que les représentations différentielles $d u$ volley-b a chez les élèves filles et garçons étaient susceptibles d'être plus finement décrites en prenant en Considération la notion d'orientation de genre, que nous avons opérationnalisée (dans le C are d'une enquête extensive) au moyen de l'IRSB. Les questions qui ont orienté l'enquête sont les suivantes :

- Comment se structurent les représentations sociales et fonctionnelles des filles et des garÇons relativement au volley-ball ?

- En quoi jouent-elles un rôle dans l'attrait ou le rejet des sports Collectifs et plus particulièrement du volley-ball en EPS ?

- Au-delà de la variable sexe n'y a-t-il pas un lien entre la variable orientation de genre et les représentations du volley-ball scolaire?

Nous faisions l'hypothèse qu'au-delà de la variable sexe, les profils représentationnels des élèves vis-àvis du volley-ball étaient susceptibles de se différenCier suivant l'orientation de genre des sujets. Nous souhaitions pouvoir caractériser par le biais d'une typologie les relations susceptibles d'exister entre Ces trois variables pour une population de lycéennes et de lycéens.

\section{MÉTHODE}

L'enquête, menée dans le cadre de l'enseignement agricole (3) avait pour but d'explorer les attitudes des élèves vis-à-vis de l'éducation physique en général et de l'enseignement en classe mixte, leurs attraits et rejets des APS enseignées et leurs représentations des sports Collectifs et du volley-ball en particulier. Nous n'exploitons ici que la partie de l'enquête relative aux liens entre trois variables: sexe, genre/IRSB, représentations du volley-ball.
Nous nous intéresserons plus particulièrement aux Contributions des variables sexe et genre/IRSB sur les profils représentationnels des élèves.

\section{Recueil des données}

Les questionnaires ont été envoyés, fin 2002 , aux neuf lycées d'enseignement général et technologique agricole publics de la région Midi-Pyrénées. Le retour de 507 questionnaires exploitables s'est échelonné entre décembre 2002 et février 2003. Les questionnaires ont été distribués en fin de cours par les enseignant/e/s d'EPS à tous leurs élèves de première quelle que soit la filière d'enseignement (taux de réponse : $100 \%$ ). L'échantillon par grappe ainsi Constitué présente une fréquence importante d'élèves préparant un baccalauréat technologique $(65,7 \%)$ et quelques élèves préparant un baccalauréat scientifique $(18,7 \%)$ ou un baccalauréat profes sionnel $(15,6 \%)$ (pourcentages usuels dans ce système d'enseignement). Il nous a permis d'avoir accès à différentes filières du second degré. Notons, par rapport à l'étude de Vouillot (2003) précédem ment Citée, que notre population ne comporte pas d'élèves engagés dans une filière littéraire. Notons aussi que la proportion de filles est plus faible (33,5\%, soit 170 élèves) que la proportion de garÇons $(66,5 \%$ soit 337 élèves). Elle est sensiblement égale à la moyenne nationale $(33 \%$ de filles sont scolarisées en lycées agricoles) établie en 2000 par les statistiques $d u$ ministère de l'Agriculture pour l'enseignement public.

Le questionnaire était constitué de trois grandes parties. La première collectait des informations sur les sujets (sexe, âge, etc. ) et comportait la passation du test traduit par Fontayne, Sarrasin \& Famose (2000) afin d'accéder aux catégories de genre/IRSB des élèves. La deuxième partie s'intéressait à l'EPS en général (utilité perçue, intérêt déclaré relativement aux différentes APS enseignées, à la mixité, au sexe $d u$ professeur, etc.). La troisième partie était Constituée de questions portant sur les sports collectifs et plus particulièrement le volley-ball. Parmi elles, deux questions exploraient les attraits et rejets des sports Collectifs et du volley-ball (Aimes-tu les sports Collectifs? Aimes-tu le volley-ball?). Deux autres questions avaient pour but d'accéder aux représentations sociales et fonctionnelles des élèves à propos du volley-ball (un test d'association de mots et un différenciateur sémantique). Nous développons Ci-après les points de méthode relatifs à I'IRSB et au recueil des représentations du volley-ball. 
Nous avons utilisé la version courte de I'IRSB dont la traduction française a été validée par Fontayne, Sarrazin \& Frose (2000) (Cf. annexe 1). Nous l'avons retenue pour plusieurs raisons: (i) le fait qu'il ait été validé pour une population d'adolescentes et adolescents français ce qui Correspond à notre popuIation de lyCéennes et lyCéens; (ii) le fait qu'il soit un outil classique en psychologie sociale du sport; (iii) sa maniabilité dans un questionnaire comprenant de multiples questions. Ce test dans sa version Courte Comprend dix-huit items, il prend appui sur l'hypothèse développée par Constantinople (1973, Citée par Hurtig \& Pichevin, 1986, p. 225-250) Considérant que masculinité et féminité sont deux dimensions indépendantes co-existantes (nous y reviendrons en disCussion). Selon Bem (1974) l'outil a pour ambition de mesurer les différents rôles attachés au genre sur la base d'une catégorisation psychologique des individus, envisagée comme indépendante du sexe biologique (Marro, 2002).

Le test d'association de mots concernant le volleyball a été adapté des tests d'association libre utilisés dans les recherches sur les représentations sociales (Abric, 1994). Il s'agit à partir d'un mot induCteur - ici, le volley-ball - de demander au sujet de produire tous les mots, expressions ou adjectifs qui lui viennent à l'esprit (dans notre étude entre cinq et sept mots au maximum). Le caractère spontané et la dimension projective du test permettent d'accéder plus facilement et rapidement que dans un entretien aux éléments qui Constituent l'univers sémantique de l'objet étudié (Abric, 1994 ; Rouquette \& Rateau, 1998).

Le différenciateur sémantique inspiré d'Osgood, Suci et Tannenbaum (1957) est une méthode d'analyse quantitative des connotations implicites et explicites associé au mot (Menahem, 1968). Elle Consiste à proposer, sur plusieurs échelles de Likert, des Couples opposés et représentatifs d'un thème (Jodelet, 1985 ; Menahem, 1968). Le différenciateur sém antique est considéré comme une échelle d'attitude prédictive d'un Comportement. Nous avons Considéré, à la suite de l'étude sur les représentations sexuées du rugby de David (1995) qu'il permettait d'approcher - par voie de questionnaire - les représentations fonctionnelles habituellement explorées en situation (Weill-Fassina, Rabardel \& Dubois, 1993). Selon David, Cet outil favorise la Construction d'une image assez lisible des représentations des sujets à condition que le choix des couples d'adjectifs ou de verbes soit adapté au cas exploré. Par ailleurs, Lorenzi-Cioldi (1988) rappelle la parenté intéressante entre la problématique de l'identité de sexe et le différenciateur sém antique (p. 132, note 24). Ainsi, pour Construire un outil pertinent d'exploration des représentations fonctionnelles $d u$ volley-ball, nous avons construit un différenciateur sémantique autour de Couples d'échelles bipolaires construites sur la base d'adjectifs et de verbes antonymes puisés dans les répertoires de l'univers lexical du volley-ball. La littérature spécialisée (notamment Cloes, Lapierre, et Pieron, 1995 ; Tanguy, 1992) montre que le volley-ball est appréhendé par les élèves selon deux pôles:

- soit le pôle Compétitif (activité d'opposition, affrontement, attaquer, rompre l'échange, faire to mber la balle dans le camp adverse, construire une attaque smashée...).

- soit le pôle récréatif (se détendre, Conservation du ballon, renvoyer, Continuité, attendre la faute de l'adversaire, Continuité de l'échange...).

Ces deux pôles sont aussi liés, dans la littérature, aux stéréotypes des rôles masculins et féminins en sports Collectifs (David, 1995 ; Griffin, 1984 ; Tanguy, 1992 ; Vigneron, 2004). Les échelles bipolaires proposées aux élèves ont été construites à partir de ces oppositions, par exemple : "rompre l'échange" contra "Continuité de l'échange" (Cf. Annexe 2 pour la présentation du test). Le différenciateur sémantique a fait l'objet d'une expérimentation préalable avec une classe de première avant d'être stabilisé.

Au terme de Cette présentation des outils de l'enquête par questionnaire, nous souhaitons souligner que les trois tests (IRSB, association de mots et différenciateur sémantique) appartiennent tous aux traditions de recherche en psychologie sociale. Par ailleurs, les deux techniques d'exploration des représentations ont été empruntées aux méthodologies classiquement en usage dans les recherches s'intéressant aux représentations. Le test d'association de mots explore prioritairement la dimension sociale des représentations, le différenciateur sémantique appréhende plus particulièrement les représentations fonctionnelles, tactiques et dynamiques, du volley-ball.

\section{Traitem ent des réponses}

Catégorisation des sujets selon les modalités de la variable genre/IRSB

Conformément aux règles énoncées par Fontayne, Sarrazin \& Famose (2000) nous avons réparti les élèves à partir des réponses au test selon les quatre modalités de la variable genre/IRSB : masculin, féminin, androgyne, et non-différencié. Leur répartition 
repose sur un partage par la médiane - median split method (Bem, 1974) qui permet d'établir les scores de la variable orientation de genre pour la population étudiée. En ce qui nous concerne, la répartition des élèves a été effectuée sur la b ae de la médiane des sous-échelles masculine et féminine de la population d'adolescent/e/s prise en référence (Fontayne, Sarazin \& Faose,2000). Rappelons que les variables orientation de genre et sexe sont indépendantes (4): un garçon, par exemple, peut être de genre féminin ou de genre androgyne. La répartition des élèves de notre échantillon est la suivante: $32 \%$ d'élèves de genre non-différencié (scores b a sur les deux sous-échelles), $26 \%$ d'élèves de genre féminin (score b a sur la sous-échelle masculine et score haut sur la sous-échelle féminine), $21 \%$ d'élèves de genre masculin (score haut sur la souséchelle masculine et score b a sur la sous-échelle féminine) et $21 \%$ d'élèves de genre androgyne (scores hauts sur les deux sous-échelles).

Catégorisation des réponses au test $d$ 'association de mots

À la question: "À quoi te fais penser le mot volleyb 由, peux-tu indiquer les mots qui te viennent à l'esprit ? ", les élèves ont produit 2354 mots (soit une moyenne de 4,7 mots par élèves) dont 523 mots différents, Crtains Cités avec des fréquences importantes. Par exemple : le mot "filet" a été Cité 201 fois, le mot "b 由on», 198 fois; le mot "smash», 175 fois; le mot "passe", 102 fois, et le mot «équipe ", 97 fois. Un premier regroupement des mots a été effectué sur la $b$ \&e de leur racine ( $p$ ar exemple, b I , b I n, etc.) et du champ lexic a évoqué par les réponses (par exemple, les mots et groupes de mots "équipe", "bonne ambiance dans l'équipe ", "équipiers", "équipe soudée ", "être en équipe ", "jeu d'équipe", etc.). Nous avons ensuite fusionné cet ensemble avec les mots relevant $d u$ même thème ("apprendre à jouer ensemble", "bon esprit de groupe", "Collectif ", "Complicité ", "Confiance en ses partenaires", "entraide", "groupe»). Nous avons donné pour titre à Cette C ágorie : "aspect collectif $d u$ volley-ball". Nous avons procédé de la même façon pour les 523 mots qui ont été ainsi progressivement regroupés en vingt $C$ ágories de "mots associés" (Cf. annexe 3) dont nous avons étudié la présence ou l'absence dans les réponses des élèves.

Catégorisation des réponses

au différenciateur sém antique

Conformément à la procédure utilisée par David (1995) une série de dix-sept oppositions séman- tiques selon des échelles de Likert en sept points a été ainsi proposée aux élèves ( $\mathrm{C} f$. Annexe 1). Nous leur avons demandé d'entourer, ligne par ligne, le Chiffre du différenciateur le plus proche de leur représentation de l'action efficace. Un premier traitement des réponses a montré que les élèves Cochaient majoritairement les extrémités de chaque échelle. Cette manière de répondre est liée, selon nous, à la logique du jeu de volley-ball, qui, comme tout sport collectif, renvoie à la $m$ ise en échec des adversaires mais aussi à l'entraide avec ses partenaires. Elle se spécifie dans la mesure où il faut à la fois empêcher que la balle tombe dans son propre camp et s'organiser pour faire tomber la balle dans le camp adverse ou amener l'adversaire à commettre une faute. Cette logique de Coopération et d'affrontement renvoie à la dynamique des actions à produire en volley-ball. Par exemple, les élèves cherchent soit la continuité du jeu ("faire durer l'échange"), soit la rupture ("rompre l'échange»). Nous avons donc traité les réponses aux échelles de Lickert comme des variables à trois modalités: les pôles opposés du différenciateur, et la zone centrale " 0 " Codée «pas de préférence".

Pour résumer, à l'issue du Codage, nous disposions d'un tableau mettant en relation 507 sujets interrogés et les variables : sexe (deux modalités); genre/IRSB (quatre modalités); attrait pour les sports collectifs (deux modalités); attrait pour le volley-ball (deux modalités); vingt variables à deux modalités de "mots associés" et dix-sept variables "différenciateur sém antique " à trois modalités.

\section{Traitem ents statistiques}

Une première étape a consisté à effectuer des tris Croisés entre les variables sexe, genre/IRSB et les réponses aux deux questions sur l'attrait ou le rejet des sports Collectifs et du volley-ball. À la question : "Aimez-vous les sports Collectifs?", la grande $\mathrm{m}$ ajorité des élèves répond : oui $(81,2 \%$ des filles et $87,5 \%$ des garçons). Le test du $\chi^{2}$ montre que malgré l'engouement des filles et des garçons pour les sports Collectifs, les garçons sont plus enthousiastes à leur sujet que ne le sont les filles (5). Les réponses à la question: "Aimez-vous le volleyball?" mettent en évidence que la très grande majorité des élèves disent aimer cette APS (72,7\% des filles et $68,2 \%$ des garçons). Mais, pour ce sport Collectif "approprié aux deux sexes", il Convient de noter - pour la population étudiée - que l'écart de préférence entre les filles et les garçons n'est pas statistiquement significatif. Les résultats, 
obtenus sur une population d'élèves des lycées agricoles sont glob ment identiques à ceux des enquêtes nationales.

À la suite de Cette première étape, deux traitements statistiques ont été utilisés pour explorer les liens entre les variables sexe, genre/IRSB, et représentations du volley-b a.

- Le premier a consisté à décrire la structure des données à partir d'une Classification hiérarchique ascendante ( $\mathrm{CAH})$. Une typologie des représentations des élèves sur le volleyball (mots associés et différenciateur sémantique) a été établie précisant le poids des modalités des variables sexe et genre/IRSB dans la définition des classes représentationnelles.

- Dans le but de saisir les implications statistiques entre les modalités des variables sexe et genre/IRSB et les structures représentationnelles. Nous avons utilisé un logiciel d'analyse implicative $m$ is $a u$ point en didactique des $\mathrm{m}$ athém atiques (Gras et al., 1996). Pour faciliter la lecture, la description des méthodes statistiques utilisées est présentée préalablement à l'exposé des résultats.

\section{RÉSULTATS : À LA RECHERCHE D.UNE RELATION ENTRE REPRÉSENTATIONS DES ÉLĖVES ET CATÉGORIES DE GENRE SELON L'IRSB.}

\section{La Construction d'une typologie des représentations Chez les lycéennes et lycéens interrogés}

Nous avons utilisé le logiciel SPSS ${ }^{\circledR}$ pour effectuer une CAH à partir des différentes modalités des variables étudiées. L'analyse des données effectue des regroupements successifs allant des individus vers le groupe et met en évidence quatre classes. Quatre profils représentationnels agrègent ainsi les réponses des élèves. Le tableau 1 condense les résultats. Chaque classe est caractérisée par les modalités des variables qui contribuent significativement à sa définition (pourcentage dans la classe). Notons qu'en ce qui Concerne les "mots associés", Certaines classes sont caractérisées, à un seuil significatif, par la modalité absence du mot associé (en italiques dans le tableau) plutôt que par sa présence, notamment la classe 2 et la classe 4 .
L'analyse des modalités de variables significativement associées à chacune des classes permet d'identifier quatre profils représentationnels :

- Classe 1: "Des garçons qui cherchent à rompre l'échange à tout prix". Il s'agit d'élèves, plutôt des garÇons (84\%), fidèles aux stéréotypes sexués " $m$ asculins" identifiés par la littérature à propos du volley-ball (modalité "attaquer» du différenciateur sémantique) qui cherchent à rompre l'échange (modalité "pas de préférence» entre précision et force).

- Classe 2: "Des garçons qui recherchent le rapport d'opposition dans un jeu collectif". Il s'agit d'élèves, plutôt des garçons $(77 \%)$, qui valorisent les représentations relatives au rapport de force (modalité fortement associée "être Combatif "), n'ayant pas "peur" de se faire mal (absence du mot "peur-douleur" significativement associée à la classe), m ais qui intègrent la notion de "jeu collectif». Il est intéressant de noter que ces élèves s'inscrivent plutôt dans une représentation du jeu en "Continuité » plutôt qu'en "rupture ", ce qui les distingue nettement des élèves de la classe précédente.

- Classe 3 : "Des élèves qui veulent progresser pour jouer et attaquer collectivement". Cette classe est construite à partir des seules modalités de la variable représentations. Aucune des modalités des deux variables sexe et genre/IRSB ne contribue de manière significative à la définition de Cette classe. On retrouve une agrégation de représentations liées à l'action qui valorise la ruse sur la force, le plaisir du jeu plutôt que la recherche du gain du match comme l'indique les items du tableau 1. Notons que le pourcentage de la catégorie "technique" des mots associés est, dans cette classe, supérieur à la moyenne.

- Classe 4 : des élèves, dont une majorité de filles, qui cherchent à maitriser le ballon pour le renvoyer. Cette classe est remarquable puisque $51 \%$ des filles y sont présentes (rappelons qu'il y a $33 \%$ de filles dans notre population). Contrairement aux trois autres classes C'est l'absence de la modalité "attaque " $(93 \%)$ qui est associée à la classe. D'une m anière générale C'est significativement l'absence de Certaines modalités des mots associés qui contribue à ıa définition de cette classe (voir les items en italiques du tableau 1). Quant aux modalités du différenciateur sémantique, ce sont plutôt les représentations stéréotypées du volley-ball 
Tableau 1. - Modalités des variables signific aivement associées ou signific aivement non a ssociées aux quatre crasses (entre parenthèses le pourcentage de la modalité dans la classe)

\begin{tabular}{|c|c|c|c|}
\hline & $\begin{array}{l}\text { V abble sujet } \\
\text { signific aive }\end{array}$ & $\begin{array}{l}\text { Les modalités } \\
\text { du différentiateur } \\
\text { sémantique }\end{array}$ & $\begin{array}{c}\text { Mots associés } \\
\text { au volley-b a } \\
\text { En italiques absence } \\
d u \text { mot associé }\end{array}$ \\
\hline Classe 1 & Sexe Garçon (84\%) & $\begin{array}{l}\text { - Pas de préférence entre précision et force } \\
(49 \%) \\
\text { - Pas de préférence entre devenir fort et } \\
\text { devenir malin }(48 \%) \\
\text { - Attaquer }(65 \%) \text { [plutôt que "renvoyer"] }\end{array}$ & $\begin{array}{l}\text { - Règlement-limites }(64 \%) \\
\text { - Attaque }(61 \%) \\
\text { - Équipement/matériel }(60 \%)\end{array}$ \\
\hline Classe 2 & Sexe Garçon (77\%) & $\begin{array}{l}\text { - Ettre Combatif (85\%) [plutôt que ne pas se } \\
\text { fairemal] } \\
\text { - Match }(70 \%) \text { [plutôt qu'entraînement] } \\
\text { - Rude }(62 \%) \text { [plutôt que doux] } \\
\text { - Progresser }(72 \%) \text { [plutôt que se détendre] } \\
\text { - Attaquer }(70 \%) \text { [plutôt que renvoyer] } \\
\text { - Risquer }(51 \%) \text { [plutôt qu'assurer] } \\
\text { - Continuité }(80 \%) \text { [plutôt que rupture] }\end{array}$ & $\begin{array}{l}\text { - Aspect ColleCtif du jeu (66\%) } \\
\text { - Peur-douleur (97\%) } \\
\text { - Règlement/limites (70\%) } \\
\text { - Équipement/matériel (73\%) }\end{array}$ \\
\hline Classe 3 & $\begin{array}{l}\text { Pas de variable sujet signifi- } \\
\text { cativem ent associée à cette } \\
\text { Classe }\end{array}$ & $\begin{array}{l}\text { - Jouer }(88 \%) \text { [plutôt que gagner] } \\
\text { - Précision (91\%) [plutôt que force] } \\
\text { - Se sentir bien (91, \%) [plutôt qu'être un/e } \\
\text { Champion/ne] } \\
\text { - Doux }(52 \%) \text { [plutôt que rude] } \\
\text { - Assurer }(68 \%) \text { [plutôt que risquer] } \\
\text { - Renvoyer (55\%) [plutôt qu'attaquer] } \\
\text { - Faire des progrès (75\%) [plutôt que devenir } \\
\text { - sportif] } \\
\text { - Devenirmalin (79\%) [plutôt que devenir fort] } \\
\text { - Se maîtriser (85\%) [plutôt qu'être le/la } \\
\text { meilleur/e] } \\
\text { - Faire durer l'échange (85 \%) [plutôt que } \\
\text { romprel'échange] }\end{array}$ & $\begin{array}{l}\text { - Règlement/limites }(77 \%) \\
\text { - Attaque }(73 \%) \\
\text { - Équipement/matériel }(70 \%) \\
\text { - Coopération }(63 \%) \\
\text { - Technique }(55 \%)\end{array}$ \\
\hline Classe 4 & Sexe Fille $(51 \%)$ & $\begin{array}{l}\text { - Regarder le ballon (71\%) [plutôt que } \\
\text { l'adversaire] } \\
\text { - Renvoyer (55 \%) [plutôt qu'attaquer] } \\
\text { - Jouer }(78 \%) \text { [plutôt que gagner] } \\
\text { - Se maîtriser (83\%) [plutôt qu'être le/la } \\
\text { meilleur/e] }\end{array}$ & $\begin{array}{l}\text { - Attaque (93\%) } \\
\text { - Règlement-limites (83\%) } \\
\text { - Technique (87\%) } \\
\text { - Rapport d'opposition (95\%) } \\
\text { - Aspect collectif jeu (62\%) } \\
\text { - Tactique }(90 \%)\end{array}$ \\
\hline
\end{tabular}

féminin telles qu'évoquées par Davisse (1991) et Tanguy (1992) qui struCturent Cette Classe, alors même qu'un grand nombre de garçons appartient à cette classe.

Ainsi, et sous différentes formes, nous pouvons conclure que seule la variable sexe participe à la définition de trois des classes représentationnelles. L'élément le plus surprenant au terme de cette analyse statistique est qu'aucune des modalités de la variable genre/IRSB n'intervient, ni ne Contribue significativement à la définition des quatre classes. L'analyse factorielle des correspondances sous jacente à la $\mathrm{CAH}$ indique que les quatre modalités de genre/IRSB (féminin, masculin, androgyne, et nondifférencié) sont situées près de l'origine des axes factoriels. Leurs Coordonnées sur les deux premiers axes de l'AFC sont les suivantes: modalité féminine $(0.00 ; 0.02)$; modalité masculine (- $0.05 ;-0.11)$, modalité androgyne $(-0.09 ; 0.14)$, modalité nondifférenciée $(0.10 ;-0.03)$. Comment interpréter Ce 
Constat, d'autant plus que la plupart des psychologues définissent l'orientation de genre en terme de processus d'intériorisation de normes, de valeurs, de traits, de $c$ aactères et de comportements attribués historiquement et socialement aux hommes et aux femmes qui modèlent les rapports de genre au monde et participent ainsi à sa représentation (Cogerino, 2005 ; Hurtig \& Pichevin, 1986 ; Marro, $2002 \& 2003$; Vouillot, 2003 ). Convaincues de l'exis tence d'une relation entre l'orientation de genre et les représentations $d u$ volley-ball, nous nous sommes tournées vers l'analyse implicative pour dépasser la description de l'existence ou non de liens entre les variables étudiées (6).

\section{Contribution des modalités de la variable genre/IRSB aux structures représentationnelles : l'apport de l'analyse implic ave}

Nous avons vu lors de l'analyse précédente que seule la variable sexe est significative de Certaines classes et qu'il y a un écrasement de la variable genre/IRSB. Nous persistons cependant à recherCher d'éventuelles interactions de cette variable avec les représentations. Pour ce faire nous avons approfondi la fouille des données et procédé à un second niveau d'analyse au moyen d'une analyse implicative effectuée par le logiciel CHIC (Classification hiérarchique implicative et cohésitive), afin d'aller voir finement s'il existe ou non des implications entre les variables sexe, genre/IRSB et les représentations du volley-ball. Le logiciel CHIC réalise des analyses implicatives, dont le principe est d'identifier dans quelle mesure telle réponse à tel item entraîne, statistiquement parlant, telle réponse à tel autre item. Il propose un graphe implicatif de variables permettant l'identification de réseaux de réponses, eux-mêmes Constitués de Chemins (Bailleul, 1995 \& 2000). Ces Chemins représentent des implications entre Certaines variables du réseau. Selon Bailleul (2000) l'analyse statistique implicative est un outil particulièrement puissant pour travailler sur les représentations et mettre en évidence leurs structures organisatrices. L'auteur souligne le caractère multidimensionnel de l'analyse implicative qui permet de dépasser le simple constat de l'existence d'une relation entre deux variables (Comme dans le cadre de l'analyse précédente) pour mettre en évidence des "réseaux porteurs de sens".

Pour mener ce second niveau d'analyse nous avons retenu les mêmes variables que précédemment auxquelles nous avons ajouté les réponses aux deux questions "Aimes-tu les sports Collectifs?" et
"Aimes-tu le volley-ball?"qui, on l'a vu, rassemblaient la plupart des élèves. Pour étudier les éventuelles implications des modalités des variables sexe et genre/IRSB aux réseaux de représentations nous les avons codées en "variables supplémentaires" (Bailleul, 2000). Ainsi Codées, Ces variables n'interviennent que dans le calcul des Contributions des catégories. Le logiciel permet de les "repérer" pour l'interprétation, notamment en indiquant leurs Contributions respectives aux différents Chemins du graphe implicatif.

\section{À la recherche des réseaux de représentations}

Le logiciel CHIC calcule les implications à des seuils qui ne doivent pas être considérés comme des indices de Corrélation entre variables, mais qui indiquent la force des implications entre elles. Plus le seuil est élevé, plus la quasi-implication entre deux variables est fiable. À titre d'exemple, au seuil le plus élevé (0.99) il n'apparaît qu'une seule implication, qui indique que les élèves qui ont répondu qu'ils n'aimaient pas le volley-ball ont donné moins de 5 mots au test d'association. Dès que nous approfondissons l'analyse, c'est-à-dire dès que nous abaissons le seuil, davantage de variables sont prises en compte dans les graphes implicatifs. Nous avons retenu, selon les Conseils des ConCepteurs du logiCiel, le seuil d'implication 0.70 en dessous duquel les interprétations en terme d'implication d'une variable sur une autre sont sujettes à discussion. À ce seuil il apparaît de très longs chemins (jusqu'à huit variables) appartenant à trois réseaux relativement bien distincts qui débouchent tous sur la modalité "j'aime les sports Collectifs". Ceci Confirme l'intérêt des élèves interrogés que les tris Croisés présentés en début des résultats avaient $m$ is en évidence. Nous présentons Ciaprès ces trois réseaux en nous intéressant plus particulièrement à la contribution des modalités des variables sexe et genre/IRSB sur quelques chemins qui les Constituent. Nous Considérons que la Contribution de ces modalités de variable est à prendre en compte dans l'interprétation à la condition que le risque - usuel dans ce type de traitement statistique de se tromper soit inférieur à 0.10 .

\section{Un réseau structuré autour de représentations exprimant l'idée qu'il faut d'abord s'entraîner, progresser pour se faire plaisir en volley-b a}

Si l'on s'intéresse aux différents chemins se finissant par: "jouer ", "se sentir bien ", "se maîtriser », "jouer Collectif " qui impliquent la variable "j'aime les sports Collectifs » il apparaît que, quelle que soit la variable en amont (Cf. schéma du réseau $A$ : "renvoyer", 
Schéma 1. - Réseau A

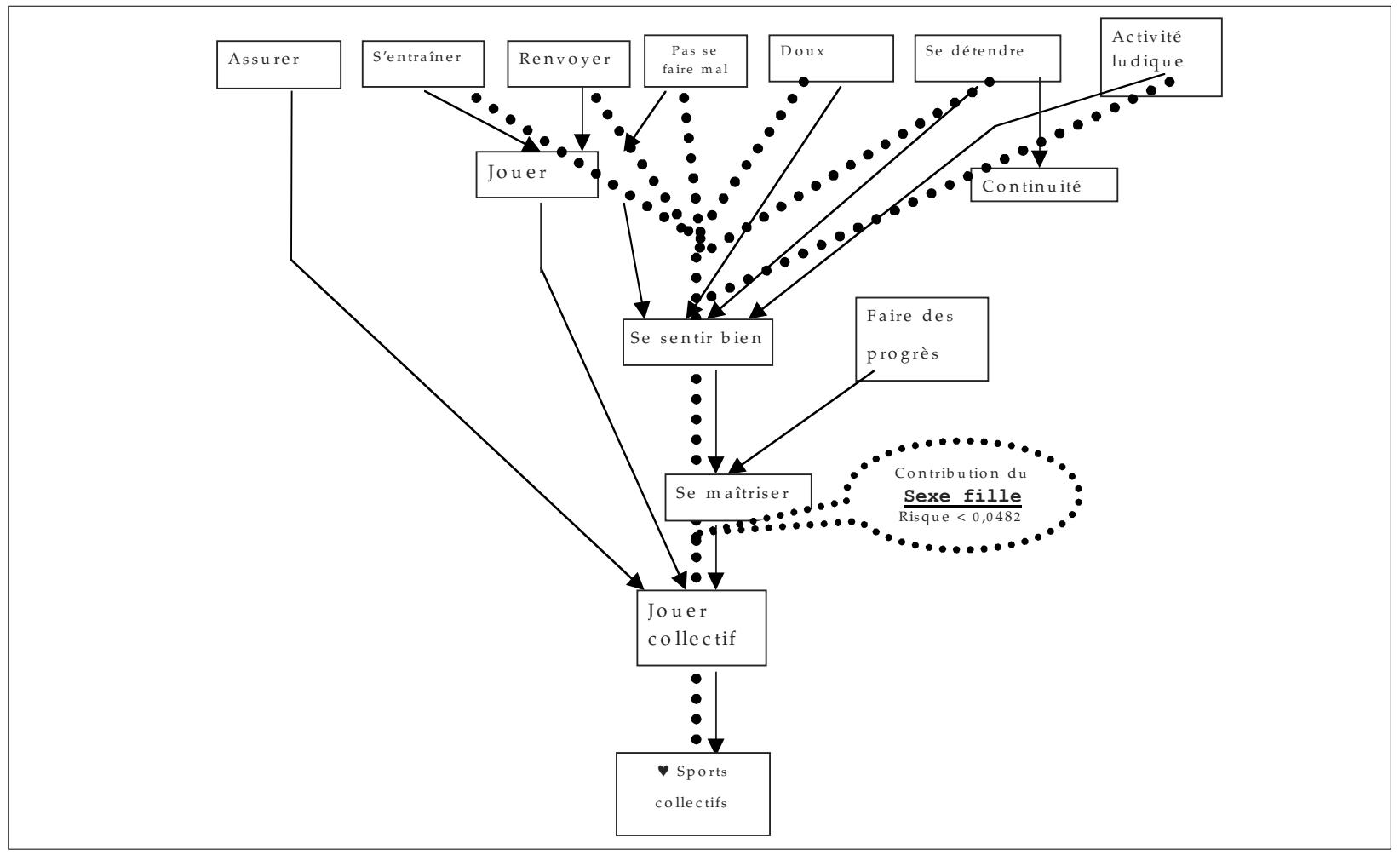

"s'entraîner ", "se détendre ", "ne pas se faire mal ", "doux"), seule la modalité fille (en pointillé sur le schém a) Contribue signific avement à Ces chemins. Sur le Chemin "activité ludique, se sentir bien, se maîtriser, jouer collectif", Cest encore la modalité fille qui Contribue signific avement. Le fait que la modalité fille de la variable sexe soit C aactéristique de la plupart des chemins de ce réseau confirme l'idée de représentations sexuées mises en évidence par de nombreux travaux didactiques autour des thèmes du jeu en équipe, du progrès et du Coté ludique de Cette activité. Ce graphe implicatif n'est pas sans relation avec la classe 4 décrite lors de l'analyse précédente. Il Correspond, selon nous, au noyau Central (Abric, 1994) des représentations que de nombreuses filles ont des sports Collectifs en général (David, 1995 ; Davisse, 1991; Tanguy, 1992 ; Vigneron, 2004).

Un réseau articulant des représentations qui soulignent la nécessité de tactiques pour jouer collectivement au volley-b a

La modalité fille (en pointillé sur le schéma) est caractéristique de seulement trois des Chemins du réseau $B$. Il est intéressant de noter que deux modalités de la variable genre/IRSB apparaissent ici significativement sur deux Chemins : sur le Chemin "qualités mentales - jeu collectif - j'aime les sports Collectifs"C'est la modalité "genre féminin" (en tirets sur le schéma du réseau $B$ ); sur le chemin "qualités physiques - j'aime le volley-ball - j'aime les sports Collectifs"C'est la modalité "genre masCulin" (tirets et deux pointillés sur le schéma). À ce seuil d'implication, il existe des Contributions spécifiques de la variable genre/IRSB relativement aux représentations du volley-ball. Ainsi les lyCéennes et les lycéens interrogés de "genre féminin"semblent partager la représentation selon laquelle le volleyball nécessite des "qualités mentales", tandis que Celles et Ceux de "genre masculin" valorisent les "qualités physiques". Ceci n'est pas sans relation avec les descriptions les plus Classiques des identités de genre et des rôles de sexe en matière de pratique des APS (Davisse \& Louveau, 1991; Fontayne, Sarrazin \& Famose, 2001 ; Vigneron, 2004). Nous pouvons émettre l'idée d'une différenCiation entre ces deux modalités du genre/IRSB rela- 
Schéma 2. - Réseau B

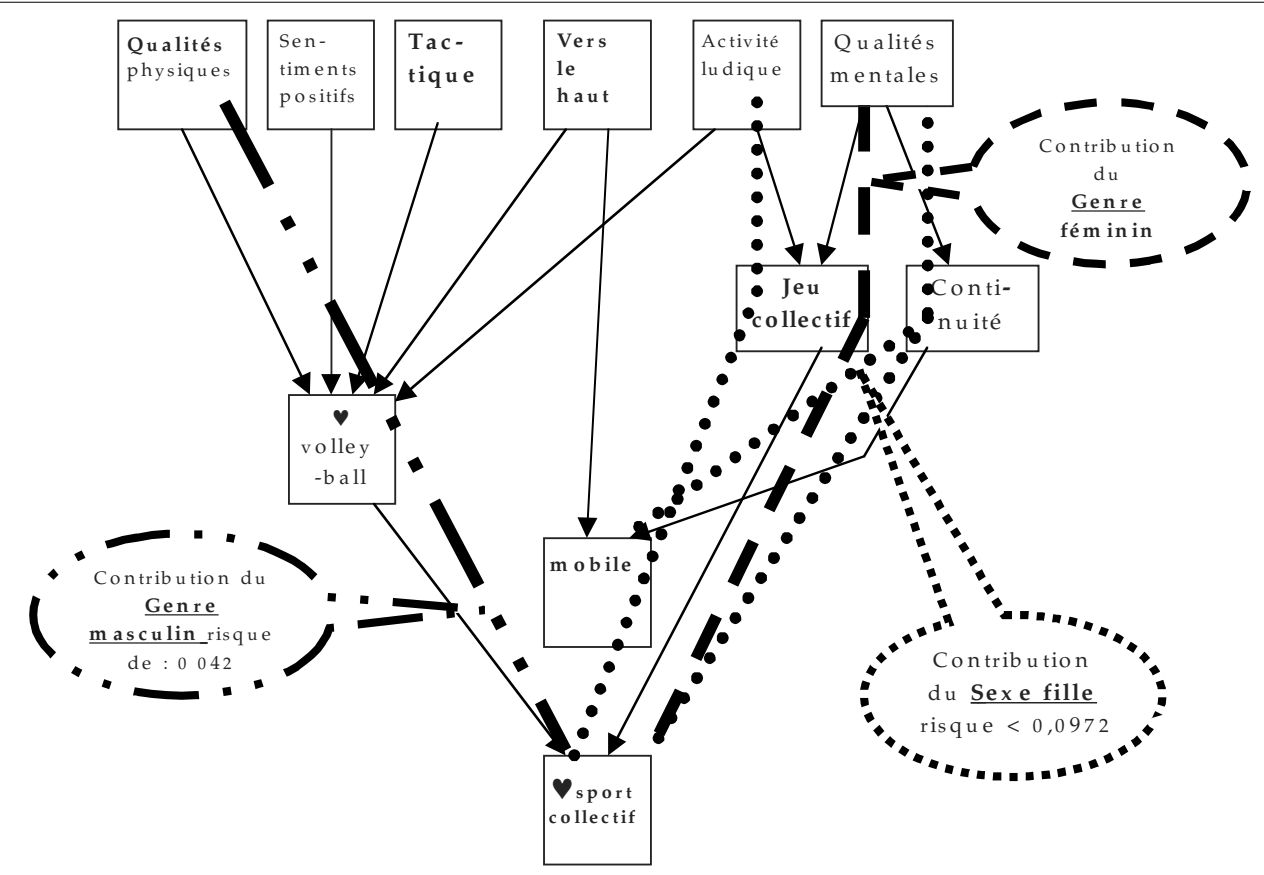

Schéma 3. - Réseau C

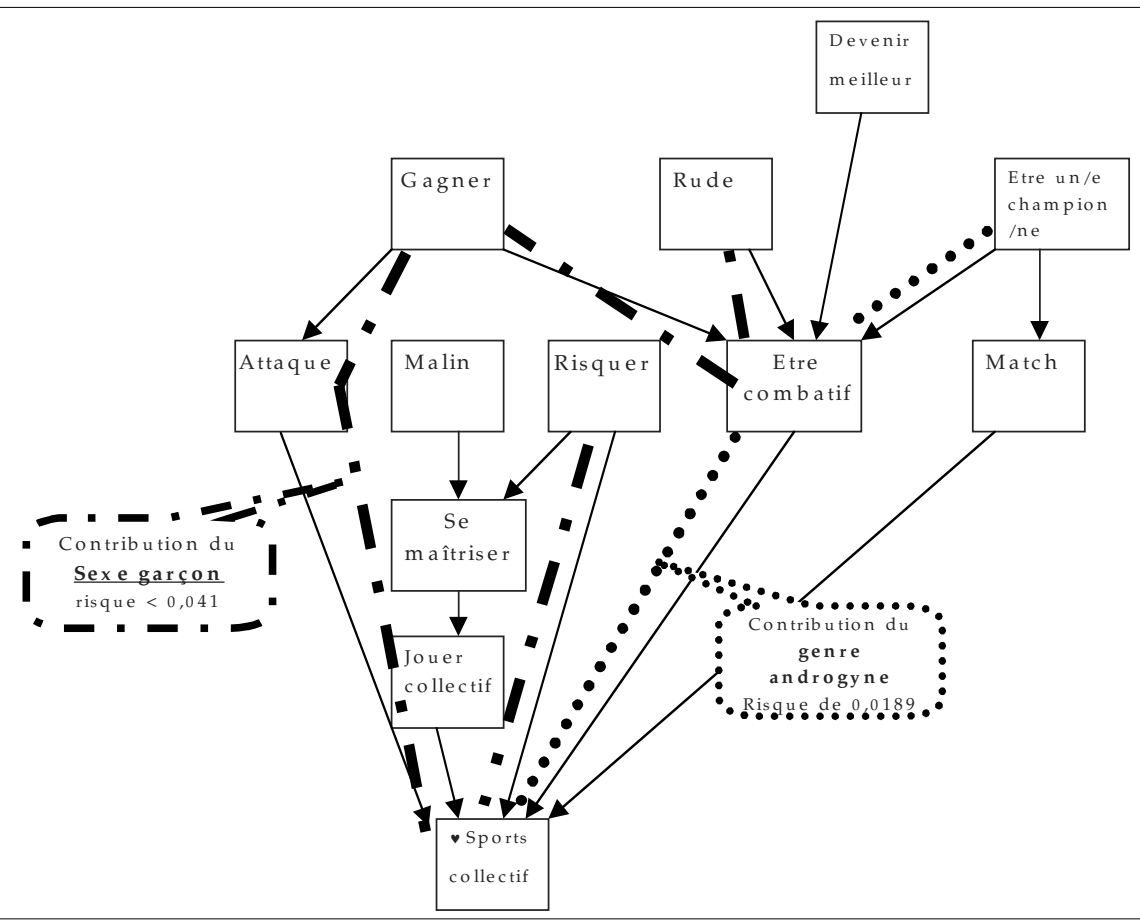


tive à l'activation des représentations du volley-b a comme jeu collectif sollicitant une activité tactique, pour les un/e/s construites par le biais de qualités physiques, pour les autres de qualités mentales au sein d'un réseau mixte, qui n'est pas sans rappeler la classe 3 de l'analyse précédente.

\section{Un réseau structuré autour de représentations $d u$ volley-b a en tant que jeu collectif d'attaque et d'opposition}

Ce réseau évoque des représentations du volleyball en tant que rapport d'opposition Collectif où l'intérêt majeur est de faire des matchs et de se mesurer à l'adversaire dans le cadre d'action d'attaques et de prises de risques. La modalité garÇon (tirets et points sur le schéma) contribue significativement à quatre Chemins de Ce réseau. Tous Ces Chemins renvoient aux stéréotypes masculins décrits dans la littérature des sports Collectifs (David, 1995 ; Griffin, 1984 ; Vigneron, 2004). Il s'apparente assez bien à la classe 2 de l'analyse précédente. Pour ce qui est du genre/IRSB, seule la modalité "androgyne" (pointillé sur le schéma), qui exprime un score élevé sur les deux échelles masculine et féminine de I'IRSB, est significative d'un Chemin : "être un/e Champion/ne, être Combatif/ve, j'aime les sports Collectifs " (7). La représentation d'adversité significativement associée aux garçons émerge dans ce réseau sous deux formes : soit de façon agressive (attaque, rude); soit de façon rusée (malin, se maîtriser, risquer).

\section{DISCUSSION}

Au terme de Ces deux analyses, que retenir? II apparaît clairement que seule la variable sexe Contribue à la définition des classes représentationnelles du volley-ball (premier niveau d'analyse) puisque les modalités de la variable genre/IRSB ne participent jam ais à la définition des quatre classes. L'analyse implicative Confirme que c'est aussi principalement la variable sexe qui - lorsqu'une de ses modalités est significativement impliquée - caractérise le graphe implicatif des différents réseaux parce qu'elle intervient sur plusieurs Chemins (notamment réseau $A$ et réseau C). Pour Ce qui est du genre/IRSB, les résultats indiquent quelques contributions des modalités "genre féminin" et "genre masculin» au sein du réseau $B$ sur deux chemins impliquant respectivement les mots associés "qualités mentales" et "qualités physiques". Marginalement, la modalité "genre androgyne " apparaît sur l'un des chemins du réseau C, réseau d'implication autour de la modalité de sexe "garçon", alors que la modalité "non différencié " (score bas sur les deux échelles de l'IRSB) n'apparaît jamais. L'étude met ainsi en évidence une participation plus significative de la variable sexe sur les différents réseaux et une participation marginale de Certaines modalités de genre/IRSB sur Certains Chemins très particuliers (8).

Ces résultats, de prime abord paradoxaux eu égard aux Conclusions des recherches sur genre et EPS (Cogerino, 2005 ; Fontayne, Sarrazin \& Famose, 2001 , 2002 ) ne sont pas sans questionner les auteures de l'article. Notre étude confirme bien qu'il existe chez les lyCéennes et lyCéens interrogés des représentations sexuées du volley-ball, alors même que ce sport Collectif est décrit par la littérature comme neutre du point de vue de ses Connotations sexuées (Fontayne, Sarrasin \& Famose, 2001). A contrario, les résultats de Ces analyses atténuent, voire contredisent, l'hypothèse initiale d'un lien fort entre les quatre modalités de genre telles qu'établies par I'IRSB et les représentations du volley-ball scolaire. Est-ce à dire que l'orientation de genre des lycéennes et des lycéens n'est pas liée à leurs représentations et que seule la variable de sexe est explicative de la diversité des représentations sociales et fonctionnelles du volleyball? C'est la discussion de Cette question que nous souhaitons maintenant aborder.

\section{Com ment interpréter le Constat d'une indépendance entre le genre tel que Construit par I'IRSB et les représentations du volley-ball?}

Notre argumentation procèdera en deux temps. À partir d'un bref recadrage anthropologique de la notion d'orientation de genre, nous reviendrons tout d'abord sur la vraisemblance d'une absence de lien entre l'orientation de genre et les représentations, Ceci nous amènera dans un second temps à discuter la pertinence de I'IRSB comme outil d'analyse.

Les anthropologues considèrent l'universalité de l'opposition féminin/m asculin (Bourdieu, 1998 ; Héritier, 1996 \& 2001 ) tout en reconnaissant la diversité des formes historiquement et Culturellement Construites et perpétuellement redéfinies des $m$ as Culinités et des féminités. Le sexe Condense les différences en tout premier lieu dans les usages corporels. L'incorporation des hexis corporelles (Bourdieu, 1998) exprime la "valence différentielle des sexes " (Héritier, 1996) Comme intériorisation des rapports de domination autour de normes et de 
valeurs traditionnellement attribuées aux femmes et aux hommes société par société. Sans prendre parti dans le déb aconceptuel autour des C ágories de sexe et d'orientation de genre, Héritier Considère "la Construction sociale du genre sous deux aspects: Comme artefact d'ordre général fondé sur la répartition sexuelle des tâches [...]; comme artefact d'ordre particulier résultant d'une série de manipulations symboliques et concrètes portant sur des individus, cette deuxième construction s'ajoutant à la première " (1996, p. 21). C'est à ce niveau nous semble-t-il que l'on peut assumer l'assertion selon laquelle la grande variété des identités de genre dans une culture donnée, se distribue dans un système d'oppositions féminin/m as Culin ne pouvant être réduit à quelques échelles, qu'elles soient uni ou bidimensionnelles comme ce fut discuté dans les années 1970 par Constantinople (1973) et Bem (1974) ou plus récemment par Lorenzi-Cioldi (1988) et Marro (2002) - Cette dernière auteure indiquant que les résultats empiriques s'avèrent insuffisants pour "fonder l'existence tangible de dimensions psychologiques pouvant être qualifiées de féminine ou masculine". L'identité de genre est, chez une personne donnée, le résultat plus ou moins stable d'un long processus qui traverse l'enfance et l'adolescence, en interaction permanente avec des normes socioculturelles qui sont elles-mêmes en évolution. Le genre renvoie, selon cette approche à laquelle nous adhérons, à la dynamique singulière de chaque être se positionnant vis-à-vis des formes stéréotypées les plus traditionnellement masculines ou féminines, avec toute la variation possible suivant les Contextes et les situations. Ainsi nous Considérerons que vis-à-vis de toute pratique sociale, dans notre cas le volley-ball scolaire, les sujets Construisent un ensemble singulier de représentations sexuées articulant des dimensions individuelles et Collectives, jouant des fonctions de Connaissance, d'orientation et de justification de l'action, susceptible de variations en contexte bien qu'ayant une Certaine Cohérence identitaire. Cette assertion élaborée sur la base des connaissances établies par différentes sciences de l'homme et de la société nous amène à ne pas invalider trop rapidement notre hypothèse initiale, à savoir qu'au-delà de la variable sexe, les orientations de genre en tant que Constructions sociales de la différence des sexes (Fraisse, 1996 ; Héritier, 1996) participent aux processus représentationnels.

Quelles explications pouvons nous alors donner aux résultats de notre enquête qui suggère une relation $m$ arginale du genre/IRSB sur les représen- tations du volley-ball exprimées par les élèves interrogés?

Une première explication consiste à pointer que les résultats portent sur des lyCéennes et lycéens de filières scientifiques, technologiques et professionnelles de lycées agricoles et qu'ils sont donc spécifiques à ce secteur du système éducatif. Sans écarter Cette hypothèse, nous pensons Cependant que les constats effectués ne reflètent $p$ as strictement des spécificités des populations d'élèves de l'enseignement agricole, dans la mesure où certains indicateurs (notamment l'attrait ou le rejet des sports Collectifs et du volley-ball) de notre échantillon sont assez similaires aux résultats d'enquêtes nationales ou d'études antérieures sur les représentations sexuées des sports Collectifs et du volley-ball (David, 1995 ; Davisse, 1991 ; Tanguy, 1992). Ajoutons que notre étude se différencie de celle de Vouillot (2003) qui porte sur les engagements sportifs extrascolaires (volontaires) des élèves et qui conclut que le sport n'est pas associé à "la féminité " Chez les lyCéennes et les lyCéens. D'une part, nous n'avons pas investigué les assignations de genre effectué par les élèves mais seulement pris en compte leur appartenance à chaque modalité établie par l'IRSB, d'autre part, nous nous sommes intéressées aux liens entre les orientations de genre et les représentations d'une APS scolaire dans le cadre de l'enseignement obligatoire. Soulignons enfin, que la répartition des élèves dans les quatre modalités de genre/IRSB ne met pas en évidence un nombre plus important d'élèves " $m$ asculins " ou "androgynes" Ce qui laisserait penser à une structuration partiCulière de la population lycéenne des filières agricoles.

Une deuxième explication invite à ne pas écarter $a$ priori l'idée que les stéréotypes de sexe sur les sports collectifs et le volley-ball sont suffisamment partagés et vivaces pour écraser toutes les variations possibles, mais marginales, susceptibles d'expliquer la diversité des représentations. Cette explication d'un primat de la variable de sexe vient Cependant buter sur la mise en évidence lors des analyses statistiques d'une classe représentationnelle non spécifiée par le sexe (Classe 2) et d'un réseau implicatif plutôt mixte (réseau B).

Les constats dont nous avons rendu Compte nous amènent à discuter la pertinence de l'outil IRSB pour Ce type d'étude. Pour ce faire nous envisageons la notion de genre dans le test de l'IRSB, ses présupposés théoriques et ses modalités de Construction pour nous interroger sur l'intérêt de ce test dans les recherches en éducation. Rappelons tout d'abord les 
éléments qui ont motivé le choix de cet outil. L'IRSB est Couramment utilisé en psychologie sociale du sport dans le C are d'études sur les processus motivationnels et les problématiques des inégalités sexuées à l'école. Le fait qu'il ait été validé auprès d'une population d'adolescents français (Fontayne, Sarrazin \& Faose,2000) était de nature à confirmer notre choix, d'autant plus que sa version courte se prêtait bien à une exploitation sous forme d'enquête par questionnaire. Par ailleurs, le projet était d'identifier les liens entre genre et représentations du volley-b $a$, thèmes d'études classiques en psychologie sociale. Nous pensions que l'utilisation de techniques en usage dans ce champ (IRSB, test d'assoCiation de mots, différenciateur sémantique) garantissait la Cohérence du projet. II n'en reste pas moins que les résultats obtenus laissent ouverte la question de la pertinence de ce choix, d'autant plus que plusieurs échelles de mesure d'orientation de genre existent (Cf. Ia revue de question de Lenny, 1991, Citée par Cogerino, 2005).

Une interprétation possible pourrait alors Considérer que I'IRSB explore les rôles très généraux ce qui ne permet pas d'accéder aux effets de genre sur un domaine très précis : la pratique scolaire du volleyball. On pourrait également dans une même ligne Critique, considérer que le volley-ball, en tant qu'APS ayant une valence neutre, faiblement sexuée a contrario du rugby par exemple, ne permet pas d'accrocher les stéréotypes féminins et $m$ asculins susceptibles d'activer de façon différentielle les diverses modalités de genre telles qu'établies par I'IRSB, tout au moins au niveau de discours provoqués par le biais des questions posées.

Sans balayer d'un revers de la m ain Ces différentes hypothèses interprétatives, nous préférons, pour des raisons que nous argumentons, nous interroger sur la fiabilité du test et plus fondamentalement Comme le suggère Marro $(2002,2003)$ à propos des différents tests se proposant à partir d'un inventaire des rôles de sexe de catégoriser les dimensions psychologiques des individus - sur "l'existence tangible de dimensions psychologiques pouvant être qualifiées de féminine ou masculine "(Marro, 2002).

Quelle est la notion de genre véhiculée par I'IRSB? L'IRSB mesure-t-il la Construction du genre au sens anthropologique que nous évoquions précédemment? Pour l'auteure de I'IRSB et ses traducteurs - le test du BSRI de Bem (1974) a été traduit en français par Hurtig et Pichevin (1986) et la version Courte validée par Fontayne, Sarrazin et Famose $(2000)$ - le test a pour ambition de saisir l'identité de genre qui peut-être variable selon les filles et les garçons. Il s'appuie sur l'hypothèse des concepts de "masculinité " et de “féminité " qui constituerait des dimensions indépendantes et non les extrémités d'une opposition, Ce qui permet de Concevoir qu'un individu puisse exprimer des dimensions à la fois $m$ asculine et féminine. Cette hypothèse d'indépendance (voire d'orthogonalité) des sous-échelles de m as Culinité et de fém inité (Constantinople, 1973, traduit dans Hurtig \& Pichevin, 1986) a été opérationnalisée par Bem, lors de la Construction du test en 1974. Il est nécessaire de souligner l'intérêt historique de remise en cause de l'échelle unidimensionnelle opposant masculinité et féminité - qualifiés par Constantinople de concepts les plus "fumeux»de la psychologie (Cité par Hurtig \& Pichevin, p. 217). La typologie de genre construite par le test a permis à l'époque d'intégrer les variations possibles de l'identité de genre tout en ouvrant un débat sur "l'androgynie psychologique " (Lorenzi-Cioldi, 1988) qui reste toujours vif. Aujourd'hui, cependant son opérationnalisation sous forme d'items très stéréotypés (9) (par exemple: "j'ai des qualités de commandement" (item masculin) et "je suis affectueux/se" (item féminin), pour ne Citer qu'un exemple de laversion Courte de Fontayne, Sarrasin et Famose (2001) n'est pas sans poser de questions (notamment discutées en psychologie par les études dévelop. pées par Marro, 2002 \& 2003 ; Vouillot, 2003). Questions épistémologiques que nous avons sans doute inconsidérément minorées au départ de notre enquête, mais qui font aujourd'hui l'objet d'une analyse Critique dans la suite de nos travaux.

Finalement et contrairement peut-être à l'ambition initiale des chercheures qui l'ont $m$ is au point, l'IRSB tel qu'il nous est parvenu reste basé sur des présupposés essentialistes. C'est-à-dire une essence $\mathrm{du}$ masculin ("la masculinité ») et du féminin ("la féminité ") immuables quel que soit le contexte. Or, Ce type d'approche débouche sur des modèles relativement statiques et stéréotypés minorant le fait que ces catégories ne sont en elles-mêmes ni universelles, ni anhistoriques. Nous référant à la posture des anthropologues (Heritier, 1996) et à la suite de plusieurs psychologues (Hurtig \& Pichevin, 1986 ; Lorenzi-Cioldi, 1988 ; Marro, 2002 ; Vouillot, 2003) nous Considérons la catégorie de genre Comme une orientation de genre, c'est-à-dire une construction sociale susceptible de varier en contexte, et qui remet en cause l'idée même des concepts de "féminité » et de " $m$ asculinité ». À partir de Ces éléments nous suggérons que I'IRSB n'est peut être pas un outil pertinent pour explorer les dimensions de genre 
dans une perspective de recherche en éduc aion qui Conjecture la possibilité d'évolution et de transformation des individus. Ceci introduit à un débat que nous laissons ouvert à la discussion, mais qui dans la suite de nos travaux (l'observation des dynamiques différentielles de Construction des savoirs en situations d'enseignement-apprentissage du volleyball) nous a amenées à considérer que l'orientation de genre doit être reconstruite en fonction des Contextes et que suivant ces derniers, diverses dynamiques sont activées marquant ainsi des " positions de genre" susceptibles de varier au fil des interactions et des situations éducatives.

Pour Conclure, il nous semble nécessaire; lorsque l'on souhaite comprendre les rapports sexués des élèves aux activités scolaires - dans notre cas, aux pratiques d'activités physiques et sportives -, de mettre en œuvre des techniques de recueil de données qui permettent de les saisir dans leur dimension diachronique. Ceci suppose des échelles d'analyse qui s'intéressent davantage aux détails à partir d'observations en classe et d'études de cas. L'indice de genre construit à travers des outils psychométriques (I'IRSB ou autres tests) nous semble devoir être $m$ is en relation avec d'autres indicateurs, si l'on veut accéder à la dynamique des couplages entre représentations, activité du sujet et situations scolaires (Schubauer-Leoni, 2002). Ceci pose aussi la question de l'articulation entre approches quantitatives et approches qualitatives. L'étude présentée ici nous semble donner matière à des réflexions à la fois théoriques et méthodologiques auxquelles nous avons modestement tenté de contribuer.

Ingrid Verscheure ingrid.verscheure@cict.fr université Paul Sabatier (Toulouse 3)

ID2-Laboratoire d'études des méthodes modernes d'enseignement (LEMME)

Chantal Am ade-Escot amade.escot@cict.fr université Paul Sabatier (Toulouse 3) ID2-Laboratoire d'études des méthodes modernes d'enseignement (LEMME)

Catherine-Marie Chiocca catherine-m arie.chiocca@educagri.fr École nationale de formation agronomique Équipe "Professionnalités, activités, travail et représentations des enseignants" (PATRE)

\section{NOTES}

(1) Dans la suite de l'article, et pour alléger le texte, nous utiliserons le terme "genre/IRSB" Chaque fois que nous ferons référence à la variable genre telle que Construite par l'IRSB (version Courte du "Bem Sex-Role Inventory", validée pour une population d'adolescents français par Fontayne, Sarrazin \& Famose, 2000).

(2) Le terme " arrière-plans représentationnels " renvoie aux travaux didactiques ayant pointé le rôle des représentations dans la façon dont est interprété, en situation, le contrat didactique par les élèves.

(3) Cette recherche a bénéficié du soutien financier $d u$ ministère $d e$ l'Agriculture et du Fond social européen dans le cadre du programme national: "Femme, formation et emploi en milieu rural "

(4) C'est l'hypothèse théorique qui préside à Ia construction du test par Bem en 1974. Dans notre population elle se Confirme puisque : $\chi^{2}$ observé $=0,23$ pour $\chi^{2}$ théorique $=7,81$ pour $3 \mathrm{ddl}$.

(5) $\chi^{2}$ observé est égal à 9,58 pour un $\chi^{2}$ théorique de 5,99 , pour $2 \mathrm{ddl}$.

(6) Signalons par ailleurs, qu'aucun $\chi^{2}$ calculé lors des tris croisés testant les liens des modalités de la variable genre/IRSB avec les réponses aux questions de la seconde partie du questionnaire (utilité perçue, intérêt déclaré relativement à la mixité, au sexe du professeur, etC.) n'ont $m$ is en évidence de liens significatifs.

(7) Cette observation est de nature à conforter selon nous un Certain nombre de Critiques faites au modèle de l'androgynie psychologique, lequel sous-estimant les effets de désirabilité sociale des rôles de sexe dominant, ne réinterroge pas le postulat de symétrie des deux sous-échelles (m asculinité et féminité) de I'IRSB, alors même que les études empiriques pointent une "propension plus élevée des hommes à l'androgynie psychologique" (Lorenzi-Cioldi, 1988, p. 155-161).

(8) Ce résultat $n$ 'est $p$ as sans relation avec celui brièvement évoqué par Fontayne, Sarrazin \& Famose (2002) dans leur article sur les effets de genre relativement aux choix et rejets des APS expliqués par "le modèle additif et différentiel de l'androgynie psychologique". Ces auteurs pointent (p. 56) que les différentes modalités de Ia variable genre/IRSB n'expliquent que $19 \%$ de la part de variance concernant le choix des APS appropriées aux deux sexes (Comme le volley-ball), Ce quiles amènent à suggérer la prise en Compte de "modèles plus complexes" sans pour autant remettre en question le modèle défendu dans leur article.

(9) Voir en annexe 1 l'ensemble des items du test.

\section{BIBLIOGRAPHIE}

ABric J.-C. (1994). Pratiques sociales et représentations. Paris: PUF.

AmADE-Escot C. (2005). "Interactions didactiques et diffiCultés d'apprentissage des filles et des garçons en EPS». In L. Talbot (dir.), Pratiques d'enseignement et difficulté d'apprentissage. Ramonville-Sainte-Agne [Haute-Garonne] : Erès, p. 61-74.
AUBERT J. (1997). Représentations sociales et comportements en gymnastique d'élèves de collège. Thèse de doctorat : sciences et techniques des activités physiques et sportives, université Joseph Fourier (Grenoble 1).

Bailleul M. (1995). "L'analyse statistique implicative: variables modales et contributions des sujets" In 
R. Gras (éd.), Actes du colloque méthodes d'analyse statistique multidimensionnelle en didactique des $m$ athém atiques. Rennes: Resses universitaires de Rennes, p.135-153.

BAILleUL M. (2000). "Mise en évidence de réseaux orientés de représentations dans deux études Concernant des enseignants stagiaires en IUFM ». In R. Gras \& M. Bailleul (éd.), Actes des journées surla fouille dans les données par la méthode d'analyse statistique implicative. Rennes: Presses universitaires de Rennes, p. 189-208.

BEM S. L. (1974). "The measurement of psychological androgyny ". Journal of Consulting and Clinical Psycho. $\log y$, vol. $42, n^{\circ} 2$, p. $155-162$.

Bergé F. (2000). "Écart de notation entre garçons et filles dans Ia Loire". In B. David (dir.), Éduc aion physique et sportive: La œrtific aion au b ac catréat. Paris : INRP, p. $97-102$.

Bourdieu P. (1998). La dom in ation m asculine. Paris : Éd. du Seuil.

BOUTHIER D. (1993). L'approche technologique en STAPS représentations et actions. Note de synthèse pour l'habilitation à diriger des recherches: sciences et techniques des activité physiques et sportives, université Paris 11 (Orsay).

BouthieR D. \& DAVID B. (1989). "Représentation et action de la représentation initiale à la représentation fonctionnelle des APS en EPS ". In G. Bui-Xuan (éd.), Méthodo. logie et didactique de l'éduc aion physique et sportive. Clermont-Ferrand: AFRAPS, p. $233-249$.

Bouthier D. ; Davisse A.; Fleurance, P. \& Lafont L. (1990), "Controverse : Les représentations en EPS". Échanges et controverses, $\mathrm{n}^{\circ} 2, \mathrm{p} .103-122$.

Clément J.-P. (1996). Représentations et conceptions en didactique: Regards croisés surles STAPS. Stras. bourg : CRDP de l'académ ie de Strasbourg.

Cloes M. : Lapierre A. \& Pieron M. (1995). "Analyse de situations d'apprentissage proposées dans un CyCle d'enseignement du volley-ball au niveau secondaire". International Volley-Tech, ${ }^{\circ} 4$, p. 9-20.

Cogérino G. (2005). "Sexe et genre en EPS". In G. Coge rino (dir.), Filles et garçons en EPS. Paris: revue EPS, p. $17-58$.

Constantinople A. (1973). "Masculinity-Fem inity: An Ex Ception to a Famous Dictum?". Psychologic \& Bulletin vol. $80, n^{\circ} 5$, p. $389-407$.

Coupey S. (1995). "Pratiques d'éducation physique et sportive au CP et différences de performances entre filles et garçons ". Revue française de pédagogie, $n^{\circ} 110$, p. $28-36$

David B. (1995). "Rugby mixte en milieu scolaire". Revue française de pédagogie, $n^{\circ} 110$, p. 51-61.

DAVID B. (2001). Éduc aion physique et sportive et évaluation: pratiques et représentations en didactique. Note de synthèse pour l'habilitation à diriger les recherches: $d$ is $\mathrm{Ci}$ pline, université Paul Sabatier (Toulouse 3).

DAvisSE A. (1999). "Elles papotent, ils gigotent: l'indésirable différence des sexes". Ville École Intégration, $n^{\circ} 116$, p. $185-198$

DAVISSE A. (2000). "EPS: Réussite des filles et mixité". In F. Vouillot (éd.), Autrement dit : filles et garçons à l'école: une égalité à construire. Paris: CNDP, p. 65-73.
Davisse A \& Louveau C. (1991). Sports, école, so ciété : la part des fem $m$ es. Paris : Actio.

Durand-Delvigne A. \& Duru-Bellat M. (1998). "Mixité scoIaire et Construction du genre". In. M. Maruani (éd.), Les nouvelles frontières de l'inégalité: hommes et femmes surle marché du travail. Paris: La Découverte, p. 83-92.

Duru-Bellat M. (1994-1995). "Filles et garçons à l'école, approches sociologiques et psycho-sociales". Revue française de pédagogie, $\mathrm{n}^{\circ} 109, \mathrm{p} .111-141$ et $\mathrm{n}^{\circ} 110$, p. $75-109$.

DUVEEN G. (1999). "Le développement des représentations sociales chez les jeunes enfants : un exemple, le genre». In M. L. Rouquette \& C. Garnier (éd.), La genèse des représentations sociales. Montréal : Éd. nouvelles, p. 114-241.

ENNIS C. D. (1998). "The Context of a Culturally unresponsive Curriculum : constructing ethnicity and gender within a contested terrain". Teaching and Teacher Educ aion, vol. 14, n 7, p. $759-760$.

Fontayne P. ; Sarrazin P. \& Famose J.-P. $(20000)$. "The Bem Sex-Role Inventory : validation of a short-version for French Teenagers". European Review of Applied PsyChology, vol. 50, n4, p. 405-416.

Fontayne P. ; Sarrazin P. \& Famose, J.-P. $(20001)$. "Les pratiques sportives des adolescents : une différenciation selon le genre". Revue STAPS, n55, p. 23-27.

Fontayne P. ; SarRazin P. \& Famose, J.-P. (2002). "Effet du genre sur le choix et le rejet des activités physiques et sportives en éducation physique : une approche additive et différentielle du modèle de l'androgynie". Sciences et Motricité, $n^{\circ} 45$, p. 45-66.

FraISSE G. (1996). La différence des sexes. Paris: PUF.

FÉLIX C. (1996). Des rapports so ciaux de sexe au savoir : une construction fém inine du rapport personnel aux mathé. matiques. Mémoire de DEA : sciences de l'éducation, université de Provence (Aix Marseille 1).

Gras R. ; Almouloud S. G. ; Bailleul M. \& Larher A. (1996). L'implication statistique : nouvelle m éthode exploratoire de données. Grenoble: Éd. La Pensée Sauvage.

GriffIN P. S. (1984). "Girls participation patterns in a middle school team sports unit ". Journal of Teaching in Physical Education, vol. 4, p. 30-38.

Heritier, F. (1996). Masculin/Fém in in : la pensée de la différence. Paris: O. Jacob.

HuRtig M.-C. \& Pichevin M.-F. (1986). La différence des sexes: questions de psychologie. Paris: Tierce.

JODELET D. [dir.] (1985). Les représentations sociales. Paris : PUF.

Marro C. (2002). "Féminité et masculinité : des modèles sociaux significatifs, mais des dimensions psycholo giques quelque peu inconsistantes". Communication, au troisième colloque international de la recherche féministe francophone. Ruptures, résistances et utopies: Toulouse, 17-22 septembre 2002.

LenNy E. (1991). "Sex Roles: the Measurement of Masculinity, Feminity and Androgyny". In J. Robinson et alii (éd.), Measures of Personality and Social Psychological Attitudes. New-York: Academ ic Press, p. 179-202.

LORENZI-CIOLDI F. (1988). Individus dominants et groupes dom inés. Grenoble: PUG. 
Marro C. (2003). "Se qualifier de "fille féminine" ou de "garçon masculin" à l'adolescence". Pratiques psychologiques, vol. $9, \mathrm{n}^{\circ} 3$, p. 5-20.

MenAHEM R. (1968). "Le différenciateur sémantique, le modèle de mesure". L'année psychologique, vol. 68 , p. $451-465$.

Mosconi N. (2003). "Rapport au savoir et division sociosexuée des savoirs à l'école". La Lettre du GRAPE, $n^{\circ} 51$, p. 31-38

Osgood C. E. ; Suci G. J. \& Tannenbaum P. H. (1957). The Measurement of Meanings. Chicago: Université of Illinois Press.

Penney D. (2002). Gender and Physical Education. London : Routledge.

SAdKeR M. \& SAdKeR D. (1991). "The is sue of gender in elementary and secondary education". Review of research in education, vol. $17, \mathrm{n}^{\circ} 7$, p. 269-334.

Rouquette M.-L. \& Rateau P. (1998). Introduction à l'étude des représentations sociales. Grenoble : Presses universitaires de Grenoble.

Schubauer-LeONI M. L. (2002). "Didactique Comparée et représentations sociales". In J.-C. Sallaberry \& G. Sensévy (Coord.), L'année de la recherche en sciences de l'éducation 2002 des représentations. Paris: AFIRSE : Matrice,p. 127149
Sensevy G. \& SallaberRy J.-C. (2002). "Représentation et didactique ". In J.-C. Sallaberry \& G. Sensévy (Coord.), L'année de la recherche en sciences de l'éducation 2002 des représentations. Paris : AFIRSE : Matrice, p. $11-17$

TANGUY G. (1992). "Le volley : un exemple de mise en $œ u$ vre didactique ". Échanges et Controverses, n 4, p. 7-20.

Verscheure I. \& AmAde-Escot C. (2004). "Dynamiques différentielles des interactions didactiques selon le genre en EPS : le cas de l'attaque en volley-ball en seconde". Revue STAPS, $\mathrm{n}^{\circ} 66$, p. 79-97.

VerscheURE I. (2005). Dynamique différentielle des interac tions didactiques et co-construction de la différence des sexes en éducation physique et sportive : le cas de l'attaque en volley-ball en lycées agricoles. Thèse de doctorat : sciences de l'éducation, université Paul Sabatier (Toulouse 3).

VIGNERON C. (2004). La construction des inégalités de réussite en EPS au baccalauréat entre filles et garçons. Thèse de doctorat: sciences de l'éducation, université de Bourgogne.

Vouillot F. (2003). "Faire des sciences et faire du sport au lycée : une logique de sexe?". L'Orientation scolaire et professionnelle, vol. $22, n^{\circ} 4$, p. 485-494.

Weill-Fassina A.; Rabardel P. \& Dubois D. [dir.] (1993). Représentations pourl'action. Toulouse : Octarès. 


\section{A nnexes}

Annexe 1. - Inventaire des rôles de sexe de Bem (traduction et validation pour des adolescents français par Fontayne, Sarazin \& Fiose, 2000)

\begin{tabular}{|c|c|c|c|c|c|c|c|}
\hline \multirow[b]{2}{*}{ je suis toujours prêt/e à écouter les autres } & \multicolumn{2}{|c|}{ Jam ais vrai } & \multicolumn{3}{|c|}{ Parfois vrai } & \multicolumn{2}{|c|}{ Toujours vrai } \\
\hline & 1 & 2 & 3 & 4 & 5 & 6 & 7 \\
\hline je suis doux/Ce & 1 & 2 & 3 & 4 & 5 & 6 & 7 \\
\hline j'ai l'esprit de compétition & 1 & 2 & 3 & 4 & 5 & 6 & 7 \\
\hline je suis sensible aux peines et aux problèmes des autres & 1 & 2 & 3 & 4 & 5 & 6 & 7 \\
\hline j'ai des qualités de commandement & 1 & 2 & 3 & 4 & 5 & 6 & 7 \\
\hline je suis affectueux/se & 1 & 2 & 3 & 4 & 5 & 6 & 7 \\
\hline je suis sûr/e de moi & 1 & 2 & 3 & 4 & 5 & 6 & 7 \\
\hline j'aime rendre service & 1 & 2 & 3 & 4 & 5 & 6 & 7 \\
\hline je suis énergique & 1 & 2 & 3 & 4 & 5 & 6 & 7 \\
\hline je suis attentif/ve aux besoins des autres & 1 & 2 & 3 & 4 & 5 & 6 & 7 \\
\hline je suis dominateur/trice & 1 & 2 & 3 & 4 & 5 & 6 & 7 \\
\hline je suis Chaleureux/se & 1 & 2 & 3 & 4 & 5 & 6 & 7 \\
\hline j'aime les enfants & 1 & 2 & 3 & 4 & 5 & 6 & 7 \\
\hline je suis sportif/ve & 1 & 2 & 3 & 4 & 5 & 6 & 7 \\
\hline je suis prêt/e à consoler les gens & 1 & 2 & 3 & 4 & 5 & 6 & 7 \\
\hline je me Comporte en Chef & 1 & 2 & 3 & 4 & 5 & 6 & 7 \\
\hline je suis tendre & 1 & 2 & 3 & 4 & 5 & 6 & 7 \\
\hline j'ai Confiance en moi & 1 & 2 & 3 & 4 & 5 & 6 & 7 \\
\hline
\end{tabular}


Annexe 2. - Le différenciateur sém antique

Voici une série de termes relatifs au volley-b a. Selon que ce qu'ils évoquent pour toi, entoure le Chiffe de Chaque ligne le plus proche du pôle qui traduit Ce que tu penses.

\begin{tabular}{|c|c|c|c|c|c|c|c|c|}
\hline Renvoyer & 3 & 2 & 1 & 0 & 1 & 2 & 3 & Attaquer \\
\hline Faire des progrès & 3 & 2 & 1 & 0 & 1 & 2 & 3 & Devenir sportif/ve \\
\hline Marquer son point & 3 & 2 & 1 & 0 & 1 & 2 & 3 & Jouer ColleCtif \\
\hline Faire durer l'échange & 3 & 2 & 1 & 0 & 1 & 2 & 3 & Rompre l'échange \\
\hline Devenir fort/e & 3 & 2 & 1 & 0 & 1 & 2 & 3 & Devenir malin/e \\
\hline$\hat{E}_{t r e}$ le/la meilleur/e & 3 & 2 & 1 & 0 & 1 & 2 & 3 & Apprendre à se m aîtriser \\
\hline Regarder le ballon & 3 & 2 & 1 & 0 & 1 & 2 & 3 & Regarder l'adversaire \\
\hline Rude & 3 & 2 & 1 & 0 & 1 & 2 & 3 & Doux \\
\hline Combatif/ve & 3 & 2 & 1 & 0 & 1 & 2 & 3 & $\mathrm{Ne} p$ as se faire mal \\
\hline Jouer & 3 & 2 & 1 & 0 & 1 & 2 & 3 & Gagner \\
\hline Etre un/e Champion/ne & 3 & 2 & 1 & 0 & 1 & 2 & 3 & Se sentir bien \\
\hline Statique & 3 & 2 & 1 & 0 & 1 & 2 & 3 & Mobile \\
\hline Progresser & 3 & 2 & 1 & 0 & 1 & 2 & 3 & Se détendre \\
\hline Entraîn em ent & 3 & 2 & 1 & 0 & 1 & 2 & 3 & Match \\
\hline Précision & 3 & 2 & 1 & 0 & 1 & 2 & 3 & ForCe \\
\hline Assurer & 3 & 2 & 1 & 0 & 1 & 2 & 3 & Risquer \\
\hline Rupture & 3 & 2 & 1 & 0 & 1 & 2 & 3 & Continuité \\
\hline
\end{tabular}


Annexe 3 - Catégorisation des "mots associés" en vingt c aégories

\begin{tabular}{|l|c|l|l|}
\hline Catégories & Oc orrence & Catégories & Oc orrence \\
\hline Activité ludique & 96 & Qualités physiques & 78 \\
\hline Aspect Collectif du jeu & 287 & Rapport d'opposition & 103 \\
\hline Attaque & 274 & Référence jeu de plage & 87 \\
\hline Coopération & 214 & Règlement/limites (notamment Filet) & 306 \\
\hline Difficile & 21 & Sentiments négatifs & 38 \\
\hline Dimension sexuée & 25 & Sentiments positifs & 49 \\
\hline Équipement/matériel (notamment Ballon) & 258 & Tactique & 99 \\
\hline Mouvement/énergie & 44 & Technique & 210 \\
\hline Peur/douleur & 55 & Vers le haut & 42 \\
\hline Qualités mentales & 44 & Non Codés & 24 \\
\hline
\end{tabular}

